\title{
Fabriquer le bonheur conjugal : sur l'argent et l'impuissance chez Tahar Ben Jelloun
}

Florina MATU, Université St. Edward's, USA

Casablanca, début des années 2000. Incapable d'exercer son art, un peintre célèbre, paralysé à la suite d'une attaque cérébrale, décide, avec l'aide d'un ami écrivain, de rédiger un manuscrit consacré à son échec conjugal et intitulé complaisamment L'homme qui aimait trop les femmes. Sa femme, bouc émissaire de tous les maux dans cette relation destructrice, trouve le manuscrit et n'hésite pas à raconter de manière beaucoup plus succincte et venimeuse sa version des faits.

Dans son roman publié en 2012, intitulé de manière ironique Le bonheur conjugal, Tahar Ben Jelloun prépare soigneusement la recette du mariage infernal. Le thème du livre se résume, affirme l'auteur dans un entretien avec le journal 20 minutes, au choc des cultures dans le mariage et l'illustration de la vérité dite par Tchekhov il y a longtemps en ces termes : «Si vous craignez la solitude, ne vous mariez pas ». Dans un autre entretien réalisé par Sarah Adida pour l'hebdomadaire La nouvelle tribune, Ben Jelloun explique combien la thématique de son roman, la crise conjugale, représente une réflexion de la crise économique et sociale que traverse le monde contemporain. L'auteur insiste sur le fait que la dynamique du mariage s'appuie sur des composantes universelles où qu'on se trouve. L'aveuglement, le manque de raison, le fait de ne pas savoir garder la distance constituent, selon Ben Jelloun, des éléments qui transcendent les frontières géographiques et qui, néanmoins, érodent les fondements d'un mariage durable. Le bonheur conjugal de longue durée, affirme l'écrivain, n'existe vraiment pas. Il faut donc posséder des talents particuliers pour le fabriquer et surtout le maintenir solide et vivant. Les failles dans cette entreprise audacieuse qu'est la construction du paradis conjugal, peuvent y apparaître à tout coin, soit qu'elles ressortent de l'intérieur-même du couple, soit qu'elles l'assaillent de l'extérieur. Ainsi, Ben Jelloun n'oublie-t-il pas d'ajouter un aspect essentiel, c'està-dire la spécificité du contexte social dans lequel évolue le couple. L'influence qu'exercent la famille et la société, s'avère capitale, insiste Ben Jelloun, surtout au Maroc. Inspirée de la vie de Picasso, la thématique du roman fait fusionner les éléments fictionnels qu'invente le romancier, de même qu'un problème social, la crise du couple dans la société marocaine contemporaine, que dissèque le citoyen Ben Jelloun. 


\section{Les facettes d'un sujet toujours d'actualité}

La démarche littéraire de l'auteur marocain n'est pas singulière dans l'espace de la création artistique. La difficulté de vivre harmonieusement dans le couple représente un sujet amplement analysé, surtout dans les œuvres cinématographiques. Un aspect crucial dans le parcours social de l'être humain, énonce le cinéaste André Cayatte, se retrouve dans la notion de solidarité du couple. Dans ses deux films de longueur identique, intitulés La vie conjugale (1964), Cayatte donne à l'homme et à la femme la possibilité de s'exprimer de manière proportionnée. Les spectateurs savourent leur rôle de complices aux deux volets de l'histoire. Il ne s'agit pas ici de se mettre du côté de Jean-Marc ou de se sensibiliser devant la perspective de Françoise, mais tout simplement de comprendre les méandres d'un couple vulnérable dont la résistance peut être minée si facilement.

Chez Ben Jelloun, la complexité de la trame narrative, doublée par les facteurs psychologiques et sociaux analysés, font que rien ne manque dans le pot au poison que constitue la vie commune des protagonistes du Bonheur conjugal. Ainsi résistent-ils au choc des classes sociales entre un homme issu d'une famille aisée et une paysanne parvenue, de la différence d'âge, de l'immixtion de la belle-famille, de la jalousie, de l'adultère, de la haine et de la vengeance. Pareils à Philippe et Odile, les protagonistes du roman Climats d'André Maurois, publié en 1928, les deux époux de l'ouvrage de Ben Jelloun unissent de manière risquée leurs différences de climats, social et comportemental, sources inévitables de frictions. Leurs tentatives d'équilibrer la tradition et la modernité dans la sphère conjugale, de faire fusionner de manière harmonieuse les traits conventionnels du mariage avec les caractéristiques de l'union moderne dont le choix libre du partenaire et le développement d'une identité féminine,

émancipée, ne réussissent vraiment pas. Ainsi, tout comme Maurois, qui se sert du récit au masculin et au féminin, l'auteur du Bonheur conjugal n'hésite-t-il pas à fouiller minutieusement dans les recoins les plus intimes de ce couple dont l'amour est progressivement empoisonné par d'innombrables malentendus.

\section{La vérité déguisée}

Pour commencer, le protagoniste prend soin de se mettre dans une lumière des plus flatteuses. Fluide dans sa profession et dans la vie privée, le peintre a su naviguer avec talent afin d'atteindre une incontestable richesse matérielle et sur le plan de ses exploits amoureux. Il est, 
selon le titre du manuscrit dans lequel il raconte sa vie, «L'homme qui aimait trop les femmes ». Ce titre est une paraphrase évidente du titre du film de François Truffaut. Tout comme Bertrand Morane, le protagoniste de L'homme qui aimait les femmes, le peintre de Ben Jelloun se vante de ses nombreuses conquêtes amoureuses, de sa virtuosité de séducteur esthète. De l'autre côté, sa femme, trahie, blessée et horriblement vexée par toutes ces amourettes, rejette pleine de mépris le titre de gloire dont s'est investi ce mari trâtre et narcissique. Il devrait s'appeler plutôt Foulane, en arabe « un individu quelconque, insiste la narratrice, puisqu'il est tellement insipide et dépourvu de qualités particulières.

Tendre et aimant? Jamais de la vie, se révolte la femme qui se lamente d'avoir vécu sous l'empire écrasant de ce conjoint menaçant, dont l'influence maléfique s'exerçait violemment et qui pénétrait impitoyablement dans les moindres recoins de son existence. Diabolique et silencieux, tel est en réalité son mari : «Ah, cette ombre omniprésente, pesante, noire, elle me suivait partout, me harcelait, se penchait sur moi et me glaçait ; elle me figeait dans mon coin » (281).

\section{Sur l'argent et l'impuissance dans le mariage}

Et pourtant, c'est surtout l'argent, doublé d'une inévitable cupidité, qui représente la source des principaux conflits dans ce ménage chaotique. Cet article se propose donc d'explorer le rôle de l'argent dans la construction du bonheur et du malheur conjugal, de même que de faire ressortir la relation disproportionnelle entre la réussite financière et l'impuissance au sens propre et figuré. Ces aspects seront analysés dans le cadre culturel marocain et français qui sert de décor au livre de Ben Jelloun, afin de faire ressortir le caractère universel des problèmes du couple dans la société contemporaine. Les deux voix narratives, masculine et féminine, s'interrogent sur la problématique du mariage moderne, de la fidélité, de l'influence exercée par les bellesfamilles implacables, victimes de leur milieu social. Quoiqu'on reconnaisse un certain souci d'objectivité de la part de l'auteur, les lecteurs remarquent inévitablement la disproportion flagrante entre les plus de deux cent cinquante pages d'un total de trois cent soixante-et-un dont dispose le narrateur, et le moins d'un tiers dont la narratrice, sa femme, se sert pour raconter sa version des faits. Il s'agit, sans doute, d'une approche intéressante de la part de l'auteur qui, tout en étant inéquitable pour ce qui est de la distribution de la sphère d'influence dans le récit, 
compense pleinement ce désavantage en doublant la véhémence et la dureté de la voix narrative féminine.

Le besoin de confession du narrateur se développe lorsqu'un accident vasculaire lui ôte presque totalement la mobilité de ses membres et le rend prisonnier dans un fauteuil roulant. Aisé, le peintre se permet d'embaucher des aides. Pourtant, leurs soins professionnels ne réussissent pas à apaiser son aigreur et une horrible et permanente sensation d'impuissance. Il ne reste pas grande chose de cet homme dont le talent artistique a toujours été doublé d'un énorme succès sur les plans amoureux et social.

La plupart des attributs de sa puissance d'autrefois, dont la force physique, l'importance sur le plan social, l'énorme potentiel artistique et la virilité, sont à présent finis ou amoindris. Les trois premières pages du roman relatent l'agacement de ce personnage sur le nez duquel s'installe, sans honte, une vulgaire mouche dont la petitesse n'empêche pas qu'elle campe souveraine et nonchalante sur le pauvre invalide. Cette scène prend graduellement des proportions hyperboliques, puisque l'auteur la décrit minutieusement, tout en faisant ressortir la rage de l'homme réduit à la merci d'un insecte : «Elle ne pèse rien, mais elle gêne. Elle énerve l'homme qui ne peut la chasser. Il a essayé de bouger, de faire du vent, il a soufflé, il a crié. La mouche est indifférente. Elle ne bronche pas. Elle est là, bien là, et ne compte pas déguerpir » (13).

Tout comme la mouche qui ouvre de manière grandiose le roman, la femme du peintre dérange, le met en colère, le pousse au bout et c'est à lui, au peintre, de trouver le moyen de triompher d'elle. Une mission difficile, d'ailleurs, puisque sa conjointe se régale de sa capacité de bousculer la vie de ce pauvre invalide, otage de sa maladie. Le moment de sa gloire est bien arrivé, affirme cette femme fière de son indépendance, de sa franchise brutale et surtout de son entêtement, ce dernier constituant un aspect fondamental de son tempérament. Tandis que l'artiste loge dans l'atelier de peinture, en attendant que sa guérison lui permette de reprendre le pinceau, sa femme occupe, sans se soucier de son mari invalide, l'autre aile de leur vaste maison de Casablanca. Elle serait, d'après le peintre, responsable de ce purgatoire qu'est devenu leur ancien paradis conjugal. Tout ce qui a contribué à l'érosion de ce mariage d'une vingtaine d'années, se trouve d'ailleurs soigneusement documenté dans le journal du mari qui, en plus, a des projets pour le publier. 
Sa carrière de peintre à succès, affirme l'auteur, s'était bâtie petit à petit grâce à un heureux mélange de talent et de discipline. A ses trente-huit ans, la reconnaissance de son travail d'artiste a été bientôt accompagnée d'un incroyable amour qui a bouleversé sa vie. Enthousiaste, il avait plongé la tête la première dans ce qui avait l'apparence d'un bonheur conjugal absolu. La chanceuse ? Une jeune femme, trop jeune et trop belle, affirment méchamment les amis sceptiques du peintre. Pour ce qui est de l'artiste, il vient de découvrir, ni plus ni moins, une perle rare, la femme idéale qu'il mérite d'ailleurs pleinement, un privilège pour lui. Il considère que son acte risqué, selon les autres, représente, par contre, la garantie d'un bonheur sans borne. Harmonieuses, les deux premières années de ce paradis conjugal sont comblées de douceur et d'une entente impeccable à laquelle contribuent équitablement les deux conjoints. D'une part, malgré sa jeunesse, la femme semble connaître les secrets d'un mélange magique d'affection, de douceur et de complaisance. Ses gestes renvoient au stéréotype de la femme soumise et pourtant satisfaite de son sort : «Elle savait le rendre bon, avait appris très vite comment s'adapter à ses manies, ses habitudes, ses lubies. Elle les acceptait avec le sourire et parfois en se moquant gentiment»(44). Cette apparence de soumission que donne la jeune épouse pendant les premières années, amène les lecteurs à croire qu'ils ont affaire à une représentation conventionnelle de la femme marocaine issue d'une famille traditionnelle, surtout si l'on prend en considération les racines paysannes de la protagoniste. On retrouve ce genre de portrait dans les études sociologiques portant sur les rapports entre les hommes et les femmes au Maroc, dont celle de Soumaya Naamane-Guessous intitulée Au-delà de toute pudeur. La sexualité au Maroc. L'auteure explique la perpétuation d'un certain comportement et d'une hiérarchie très claire au sein du foyer marocain : «La fillette est mise très tôt au service de l'homme, et à partir de ce moment-là s'établit une position d'infériorité dans laquelle la femme reste figée toute sa vie, et dans laquelle elle se cantonne elle-même poussée par sa propre mère » (17).

Dans le roman Le bonheur conjugal, le rapport de forces dans le foyer s'avère être plutôt équilibré dans le contexte socioculturel du Maroc contemporain. La femme ne semble guère condamnée à une position de subordination. Sa douceur et sa flexibilité caressante sont pleinement récompensées, ajoute l'auteur, puisque, de son côté, le mari lui rend ses manifestations d'amour en l'emmenant avec lui dans des voyages à travers le monde lorsqu'il a des expositions à des foires d'art, tout en lui ouvrant généreusement la bourse pour qu'elle achète tout ce qui lui plait. Elle n'oublie pas d'adjoindre des cadeaux pour sa famille. Ah, la famille, la 
belle-famille, un sujet épineux dans tout ménage, y compris celui du peintre, tout paradisiaque qu'il fut. La fête de mariage dont tout couple se souvient avec tendresse comme du plus beau jour de leur vie, s'avère un véritable cataclysme lorsque les deux familles se rencontrent pareilles à «deux blocs que rien n'avait pu rapprocher » en provoquant un inévitable choc des classes sociales (57). Baignée de larmes, la jeune épouse se remémore ce jour triste et sombre où elle avait quitté sa famille et s'était condamnée à une union qu'elle compare ni plus ni moins à « une descente aux enfers insoupçonnable » (292). Habits, d'une part, luxueux, d'autre part, modestes, regards et gestes crispés, distance hostile, silences gênés, accusateurs, tout semble creuser de plus en plus profondément le gouffre qui séparent les deux clans, dont le rang social et le pouvoir financier sont diamétralement opposés. Pour commencer, les parents de la mariée s'étaient saigné aux quatre veines afin de louer une belle salle de fête à Casablanca, un geste d'ailleurs reçu avec des moues de mépris par les parents du peintre, scandalisés de voir leur fils se marier dans ce décor propice plutôt aux immigrés de retour au pays. Malheureuse et pourtant profondément amoureuse, la protagoniste avoue avoir ignoré tous les signes annonciateurs de cette aventure périlleuse débutant sous de mauvais augures lorsqu'elle avait signé d'une main tremblante l'acte qui scellait son esclavage et toute une vie ombragée par des humiliations. C'est à la tante du peintre, une dame de la haute bourgeoisie de Fès, de cracher à haute voix le mépris pour cette mésalliance illustrée par la future belle-famille de son neveu, ces vulgaires campagnards indignes de respirer le même air d'élégance et de noblesse. Quant à la mariée, ils ne lui restent que la douleur et les larmes d'impuissance, de même que « les souvenirs des insupportables humiliations dont elle avait été victime dans son enfance parce qu'elle était de condition modeste [qui] lui revenaient comme une blessure secrète qui se rouvrait d'un coup » (60-61).

On y ajoute le fait que toute petite, son père l'avait vendue pour une liasse de billets à des Français aisés, un souvenir déchirant, source d'un complexe d'infériorité ineffaçable. Dure, sèche, impitoyable, tout comme le bled poussiéreux de son enfance, la narratrice évoque avec amertume l'environnement où elle a passé les premières années de son enfance, ce «village du néant », « un cimetière habité par des vivants », « une terre pourrie » où rien ne pousse, surtout pas la gentillesse et la chaleur (265). Les souvenirs de son enfance comprennent des escapades à l'école du village, loin des yeux d'un père qui voulait faire de cette petite assoiffée de lectures une bergère, suivies par l'exploitation et la famine chez son oncle Boualem, épicier avare de 
Marrakech, pour culminer avec un épisode déchirant, source d'un complexe d'infériorité ineffaçable. Une paysanne pauvre, apprend la petite, ne vaut pas grande chose. Tout comme une marchandise de valeur douteuse, on peut la vendre contre une liasse de billets, comme l'a fait son père. Les bénéficiaires de cet échange bon marché ? Un couple de Français, gentils, généreux, confiants dans la capacité de la petite d'apprendre, de sortir de l'ignorance à laquelle elle était condamnée. Des études de lycée honorables, suivies d'un diplôme de bac obtenu de justesse et une carrière prometteuse de mannequin en France, n'ont jamais pu effacer la blessure que lui avait infligée le geste du père. Un geste conservé dans les tréfonds de sa mémoire, un rappel constant de sa valeur indéterminée en tant qu'être humain.

\section{L'argent et son rôle dans les foyers marocains}

Les lecteurs ne seront donc pas surpris de constater que l'argent, de même que son rôle dans la corrosion du bonheur conjugal, préoccupe de manière évidente la femme du peintre qui le traite dans un chapitre à part. Présent dans ce contexte fictionnel chez Ben Jelloun, l'argent se situe néanmoins au cœur des discussions quotidiennes des femmes marocaines. Les études sociologiques dont celle de Hakima Mounir, intitulée Entre ici et là-bas : le pouvoir des femmes dans les familles maghrébines insistent sur l'importance de cet aspect de la vie quotidienne et de l'impact qu'il a sur les rapports entre le mari et la femme : "Même avant le mariage, les mères conseillent à leur fils de faire attention à leur argent en leur disant que certaines femmes considèrent un homme comme un poulet et elles le déplument ou une vache à lait ou alors que les femmes ne regardent que la poche ; c'est-à-dire si elle est pleine, le prétendant est intéressant, tandis que dans le cas contraire, la femme cherche une autre bourse » (86). Il semble donc que ces constats se retrouvent dans l'univers romanesque de Ben Jelloun.

Compliqué et douloureux, le sujet sur l'argent provoque invariablement la colère du mari, affirme la narratrice. Elle n'hésite pas à coller sur son conjoint toutes sortes d'épithètes, de l'inoffensif « économe » aux plus sévères appellatifs, dont «avare », « mesquin », et « radin ». Ce grippe-sou, avoue la narratrice, a tout carrément condamné sa femme à une vie qui se résumait au strict nécessaire et aux soldes, jamais au superflu et au luxe, tout en la traitant comme une gamine irresponsable. La prospérité du ménage n'était guère doublée d'une générosité naturelle, un aspect qui fait ressortir la rage de la narratrice dont les accusations tombent violemment sous la forme d'une tempête de phrases courtes et bien perçantes suivies 
d'une tirade trempée de colère : «Il compte tout. Rien n'est dépensé au hasard. Dans son cœur il y a une calculatrice. Il m'accuse d'être une consommatrice compulsive, quelqu'un qui ne fait pas de différence entre les billets, qui croit qu'une carte de crédit est un puits sans fond, que de toute façon ayant peu travaillé, je ne connais pas la valeur de l'argent et surtout que je n'ai jamais appris à compter » (305).

Et pourtant, tandis qu'elle accuse son mari de mesquinerie, Amina affiche de son côté une passion semblable pour l'argent. Elle poursuit avec férocité et habileté les tours de passepasse financiers de son malhonnête conjoint, ses comptes bancaires à Gibraltar, toutes sortes de biens acquis en Europe et même un coffre verrouillé où l'infâme avait caché de l'argent, des bijoux et des actes d'achat et de vente que sa femme n'hésite pas à copier. Munie de ces documents-dynamite, elle se réjouit en faisant des scénarios où une simple lettre au fisc marocain suffirait pour jeter son mari en prison, faible consolation, car le choc de cette nouvelle duplicité la rend ébahie, en proie aux doutes et aux questions lourdes, sans réponse : "Comment vivre à côté d'un homme qui a tant de secrets? Comment avaler cette double ou triple vie ? Côté trahison conjugale, j'étais servie depuis longtemps, il ne manquait plus que de découvrir le secret de ses activités économiques » (306).

Cette injuste avarice n'est pourtant pas généralisée, précise la narratrice. Le peintre déborde de générosité quand il s'agit de sa famille, surtout une sœur un peu sorcière, d'après Amina, qui, d'ailleurs, lui aurait jeté un mauvais sort. Profiteurs, les membres du clan de son mari vivent tout bonnement sur son dos, explique cette femme vexée tout en l'accusant de faiblesse, de naïveté et de s'être toujours fait avoir par le premier venu. Elle y voit sans doute une conspiration universelle, un vol incessant dont profitent aussi les amis et ses agents, tout un tas d'escrocs qui lui préparent coup après coup. Il s'agit ici, insiste Amina, d'un vrai trouble psychologique, qui a besoin d'être traité avec soin, comme toute maladie qui échappe au contrôle des non-spécialistes : «Tu as un sérieux problème avec l'argent, tu devrais te faire soigner » (309).

On comprend donc le fait que l'argent représente la raison des principaux conflits dans ce couple en désamour. Source de colère et de frustration, l'argent ou plutôt sa distribution inéquitable, selon la narratrice, la pousse à des gestes extrêmes. L'idée de voler un des tableaux du mari la séduit mais, hélas, aucun n'était terminé, surtout qu'il les signait à la dernière minute. Pas de chance, pas comme les épouses de leurs amis artistes qui, souligne la femme dépitée, 
habiles et souveraines, ramassaient le pognon sous les yeux dociles de leurs maris. La situation est grave, insiste la femme, il faut absolument y résister à tout prix. Il s'agit d'une véritable crise, une question de vie et de mort, car cet argent offert si généreusement aux autres, garantit tout comme le sang qui coule dans ses veines, son existence-même: "Il fallait réagir, arrêter l'hémorragie » (308). A la guerre, comme à la guerre, tout est permis, il faut développer des stratagèmes des plus raffinés. Comme le vol des tableaux n'était pas possible, la femme réclame de l'argent en surgissant dans l'atelier du conjoint au beau milieu de ses élans créateurs, sachant que l'artiste signerait des chèques sans trop y faire attention, juste pour avoir la paix. Le geste de donner de l'argent selon la demande et la nécessité représente d'ailleurs une pratique habituelle dans la famille marocaine, souligne Mounir, qui y voit «une façon pour le mari de gérer et de garder le contrôle sur son argent, une manière de ne pas céder son pouvoir à l'épouse » (95). Une fois ce pouvoir monopolisé, explique la chercheuse, «il est difficile de le lui confisquer, notamment quand la femme s'avère bonne gestionnaire » (95).

Dans le roman Le bonheur conjugal, il est évident que la femme ne risque pas de s'emparer de ce pouvoir. Cependant, elle peut bien profiter des moments de faiblesse de son mari, afin de se constituer un «trésor de guerre ». Un jour d'aumône lorsque la vigilance du peintre se trouvait au plus bas, la chanceuse s'empare d'un chèque qui ne portait que la signature de son mari, un chèque en blanc. L'extase de la femme confirme son amour démesuré pour cet argent qu'on lui a tant refusé et qu'elle touche par un coup de chance inespéré. En route vers la banque, grisée de bonheur, elle éprouve enfin un sentiment de satisfaction qui lui avait été refusé pendant si longtemps : «J'étais folle de joie. Je pouvais dévaliser son compte » (312). Elle chante cette victoire qui la venge enfin de son perpétuelle humiliation : «Je me sentais légère car mon sac était lourd de pognon, de son pognon !» (312)

\section{L'argent et l'écroulement du bonheur conjugal}

Les autres chapitres dans lesquels Amina verse son trop plein de malheur conjugal, incluent d'autres aspects cruciaux d'un ménage, dont la belle-famille, le sexe et la jalousie. Il y en a qui débordent de sarcasme, dont celui où la narratrice résume les défauts de son mari, tout en le définissant dans des phrases courtes et perçantes. On remarque dans sa tirade les deux définitions suivantes : «Mon mari est au fond un vieux célibataire, maniaque et égoïste » et «Mon mari est naïf, faible et sans autorité » (340). Du sarcasme à la haine, il n'y a qu'un tout 
petit pas. Des définitions au caractère informatif mentionnées ci-dessus, la narratrice passe au niveau suivant, tout en faisant jaillir sa haine, explosive et hideuse: «Je déteste les bourrelets autour de sa taille»(348). Elle devient plus perçante en affirmant : «Je déteste sa violence muette, son indifférence, ses absences » (348). Elle est vraiment formidable, confirme l'auteur dans un entretien avec Amobe Mevegue sur France 24, elle ne laisse rien de côté, tout en justifiant l'intensité de sa haine par la présence d'une blessure profonde chez ce personnage féminin.

La fin de cette réponse venimeuse au manuscrit du peintre ne semble pas précipiter ce couple dans le gouffre du malheur éternel. Tandis que le mari ne voit d'autre issue à leur duel marital qu'un silence et un manque total de réaction, la femme stupéfie tout le monde en déposant les armes. Par un coup de théâtre que personne ne devinait, Amina décide de lui rendre tout le malheur qu'il lui a fait avaler pendant de longues années, en le comblant de bonté, de douceur, de générosité et surtout d'humilité dont on ne la croit guère capable. Une fin heureuse, s'imaginent les lecteurs qui seront tentés de s'accrocher-à ce rayon de soleil. C'est sans compter, évidemment, sur la vraie nature de cette femme dont le plan diabolique détruit nos derniers espoirs : «Je ferai tout pour qu'il soit ma chose, mon objet, mon malade, totalement, entièrement dépendant de moi et rien que de moi » (361).

Sinueux et troublant, tel est le sort du soi-disant bonheur conjugal décrit par Ben Jelloun dans son roman consacré à la dynamique du couple contemporain, un thème universel vivement analysé et toujours d'actualité. Raconter son parcours marital au masculin et au féminin s'avère une tâche délicate, inévitablement subjective, qui alimente la frustration et la rancœur des deux protagonistes. Comment le couple contemporain peut-il donc résister aux assauts des éléments extérieurs ? Traumatisme remontant à l'enfance malheureuse, estime de soi brisée en morceaux, valeurs familiales diamétralement opposées, l'argent en tant qu'aspect (dé)valorisant de l'individu, le tout conspire contre la fragilité du bonheur conjugal dans une société qui s’appuie sur des structures précaires.

\section{Bibliographie}

Adida, Sarah. «Quand Tahar Ben Jelloun dissèque le bonheur conjugal ». La nouvelle tribune (2012). http://lnt.ma/tahar-benjelloun-et-le-bonheur-conjugal/ [consultée le 15/07/2014]

Ben Jelloun, Tahar. Le bonheur conjugal. Paris : Gallimard, 2012. 
Brion, Clo. «Entretien avec Tahar Ben Jelloun». 20 minutes (2012) http://www.20minutes.fr/livres/994587-bonheur-conjugal-tahar-ben-jelloun-chezgallimard-paris-France [consulté le 20/02/2014]

Cayatte, André. La vie conjugale. S.1 : s.n., 1964. Archives.

Maurois, André. Climats. Paris : Calmann-Lévy, 1928.

Mounir, Hakima. Entre ici et là-bas : Le pouvoir des femmes dans les familles maghrébines. Rennes : PU de Rennes, 2013.

Naamane-Guessous, Soumaya. Au-delà de toute pudeur : La sexualité féminine au Maroc : Conclusion d'une enquête sociologique menée de 1981 à 1984 à Casablanca. Casablanca : SODEN, 1987.

Truffaut, François, Michel Fermaud, Suzanne Schiffman, Charles Denner, Brigitte Fossey, Nelly Borgeaud, Geneviève Fontanel, Leslie Caron, Nathalie Baye etJean Dasté. L'homme qui aimait les femmes. Santa Monica, CA : MGM Home Entertainment, 2001. 